\title{
Demonstration of a Portable HTS MMIC Microwave Receiver Front-end
}

\author{
Jia Du, Jia Wang, Ting Zhang, Dandan Bai, Yingjie Jay Guo, Fellow, IEEE, and Yusheng He,
}

\begin{abstract}
We report the first demonstration of a portable HTS monolithic microwave integrated circuit (MMIC) receiver front-end module operating on a commercial mini cryocooler. The HTS circuit consists of a step-edge junction mixer and a number of HTS filters fabricated on a single MgO substrate. The HTS MMIC circuit is integrated with the mini cryocooler. The sample vacuum chamber, cold-head, compressor and cooling fans are all packed into one customer-designed portable box of approximately $350 \mathrm{~mm} \times 350 \mathrm{~mm} \times 250 \mathrm{~mm}$ in dimension. The HTS Josephson junction-based microwave circuit operated successfully in the cryocooler unshielded without observable performance degradation. The design and implementation of the compact unit and performance evaluation of a HTS MMIC frequency down-converter are presented.
\end{abstract}

Index Terms-High-temperature superconductor, Josephson mixer, receiver front-end, mini cryocooler.

\section{INTRODUCTION}

$\mathrm{H}$ IGH temperature superconducting (HTS) thin-films have ultra-low surface resistance values at microwave frequencies, which have been applied to make high-Q resonators and "super filters" with superior performance [1-4]. Novel nonlinear high-frequency devices, such as oscillators and mixers [5-8], based on the low-noise Josephson junctions have also been developed and successfully integrated on-chip with HTS filters recently [9-11]. These microwave filters and Josephson junction devices are mainly being developed for wireless communication front-end receiver systems. Although significant progress has been made in recent years and some filters have been successfully deployed in commercial receiver front-end subsystems, the HTS receiver systems have not been widely adopted by industry due to their two drawbacks as below. The first one is the need of cooling either by cryogens (liquid nitrogen or liquid helium) or electronic-powered cryocoolers. Implementation of superconducting electronic devices in HTS technology, instead of the low temperature superconductor (LTS) technology offers the potential to use

Manuscript received August 12, 2014.

J. Du is with CSIRO Future Manufacturing Flagship, PO Box 218, Lindfield, NSW 2070, Australia, phone: 61-2-9413 7107; e-mail: Jia.Du@csiro.au.

J. Wang, D. D. Bai, and Y. S. He are with Institute of Physics, Chinese Academy of Sciences, Beijing 100190, China, e-mail: wangjia.s1@163.com; dandanbai1008@126.com; yshe@aphy.iphy.ac.cn.

T. Zhang is with CSIRO Digital Productivity \& Services Flagship, PO Box 76, Epping, NSW 1710, Australia, e-mail: Ting.Zhang@csiro.au; Jay.Guo@csiro.au.

Y. Jay Guo is with University of Technology, Sydney (UTS), Ultimo, NSW 2007, Australia, e-mail: Jay.Guo@uts.edu.au. much cheaper and more compact cooling systems. HTS filters have been successfully developed using advanced one-stage mini cryocoolers, but rarely any demonstration of HTS Josephson junction-based active devices operating on these mini-cryocoolers due to their extreme sensitivity to mechanical vibration and electromagnetic interference (EMI) noise introduced by the mechanical coolers. Oppenlaender $e t$ al. successfully operated HTS array superconducting quantum interference filters (SQIFs) in a commercial miniature cryocooler [12] owing to a large dynamic range of these devices. The second drawback is that most HTS front-end receiver systems employ a superconductor-semiconductor hybrid configuration, i.e. using superconductor passive components combined with semiconductor active devices. This is because HTS Josephson junction-based active devices are far less developed than those of their LTS counterparts. This compromise makes the front-end bulky, inefficient and unable to exploit the full potential of the HTS superconductive components, results in the need of higher cooling power. A novel superconducting receiver system comprising both HTS passive and active devices is a much desirable technology. It can potentially improve the performance of present microwave receivers and reduce the cooling power required.

In this paper, we report the successful demonstration of a HTS monolithic microwave integrated circuit (MMIC) frequency down-converter integrated with a commercial mini cryocooler (LIHAN model TC4187B). The design and implementation of the compact unit and the performance evaluation of the HTS down-converter module are presented. To the best of our knowledge, this is the first demonstration of a portable HTS monolithic microwave integrated circuit receiver system operating on a commercial single-stage mini cryocooler.

\section{HTS MMIC DEVICE DESCRIPTION}

We have recently developed an all-HTS MMIC Josephson down-converter module [11] which consists of a step-edge Josephson junction mixer, a 10-12 GHz bandpass filter [3] for RF signal input, a lowpass filter [13] for the intermediate frequency (IF) output and a resonant strip line for local oscillator (LO) isolation. All the components are integrated onto a single $10 \mathrm{~mm} \times 20 \mathrm{~mm} \mathrm{YBa}_{2} \mathrm{Cu}_{3} \mathrm{O}_{7-\mathrm{x}}(\mathrm{YBCO})$ film on $\mathrm{MgO}$ substrate. The HTS MMIC chip was fabricated using the CSIRO-developed step-edge junction technology [14, 15]. A packaged 10-12 GHz HTS MMIC down-converter chip is shown in Fig. 1. A PCB below the MMIC chip contains RF blocking network and DC biasing lines where $1 \mathrm{k} \Omega$ resistors 
were inserted. The total size of the packaged MMIC downconverter module is approximately $25 \times 27 \times 15 \mathrm{~mm}^{3}$, which is very compact.

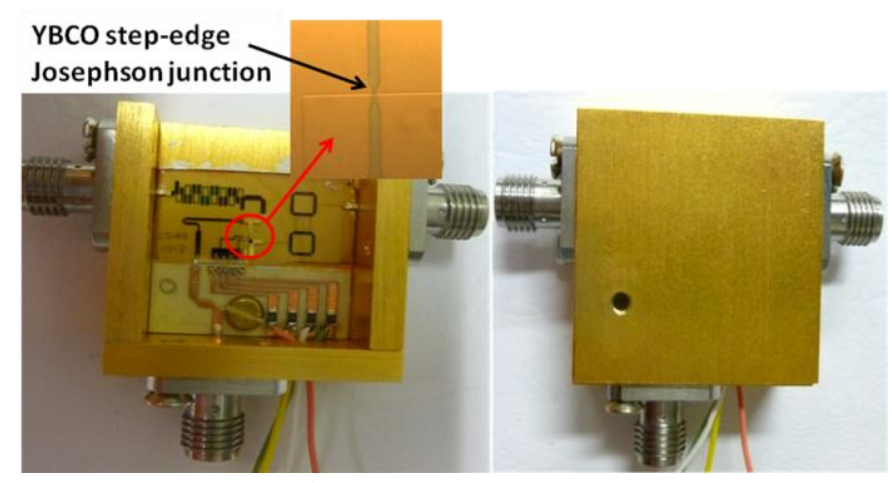

Fig. 1. Photographs of a packaged 10-12 GHz HTS MMIC Josephson frequency down-converter; the inset shows the close view of the step-edge Josephson junction.

The DC characteristics and RF performance of an optimized HTS MMIC down-converter of the same design have been fully characterized at temperatures ranging from 20 to $80 \mathrm{~K}$ on a large two-stage pulse-tube cryocooler (PTC) where the compressor is located outside the laboratory. The detailed design considerations and the mixer performance evaluation of the HTS MMIC circuit can be found elsewhere [11]. In this work, we focused on the design and integration of the HTS device with a commercial single-stage mini cooler into a single box to form a portable HTS MMIC receiver submodule. We have evaluated the performance of the HTS Josephson down-converter module to demonstrate the feasibility of a portable HTS MMIC microwave receiver frontend.

\section{MINI CRYOCOOLER AND SYSTEM INTEGRATION}

The commercial mini cryocooler (Model CT4187B) was supplied by LIHAN Thermoacoustic Technologies Co. (www.lihantech.com), China. It is a modified split type pulsetube cryocooler and has a cooling power of $10 \mathrm{~W}$ at $80 \mathrm{~K}$. It reaches $70 \mathrm{~K}$ in about 30 minutes and the lowest attainable temperature is $\sim 55 \mathrm{~K}$ after installation of all the cables and wires. The orientation of the split compressor and the cold finger was customer designed and implemented by LIHAN as shown in Fig. 2(a). It was designed in such a way that the anticipated magnetic flux generated by the compressor is parallel to the HTS chip surface so the step-edge junction would see minimum vertical flux. An instrument box was designed to accommodate all the parts including the compressor, cold finger, cooler power control circuits, fans and the sample vacuuming chamber as shown in Fig. 2 (b). Stainless steel plates were placed between the main parts to provide further isolation between the cold-head and the compressor and the power control unit. Fig. 2 (c) shows the customer-designed (built by LIHAN) vacuum chamber for housing the sample on the cold finger with vacuuming valve and the panels for cable and DC wire connections.

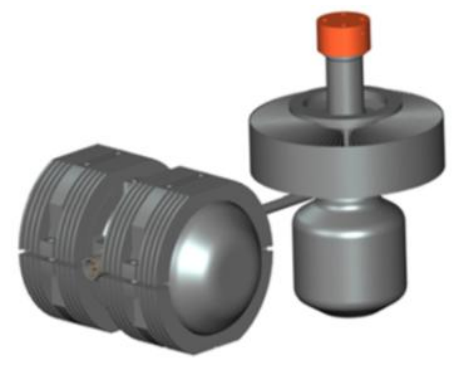

(a)

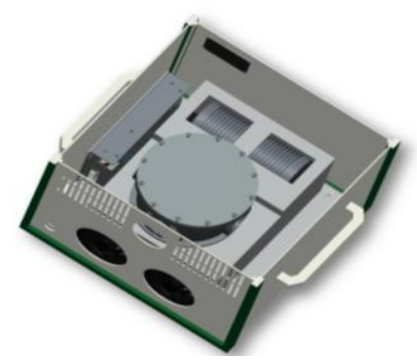

(b)

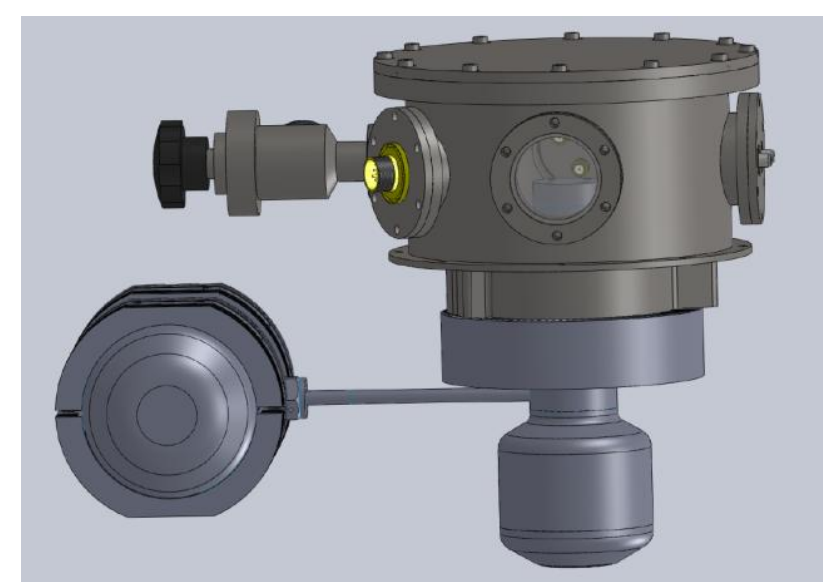

(c)

Fig. 2. Schematic diagrams of (a) LIHAN model CT4187B split-type mini cryocooler; (b) a housing box for all parts - compressor, cold finger, sample vacuuming chamber, cooler control circuit and fans; (c) sample vacuum chamber integrated with the coldfinger.

Fig. 3 (a) shows the photographs of the mounted HTS MMIC Josephson down-converter on the cold finger inside the vacuum chamber. A $\mathrm{Cu}$ sample plate was used to thermally archor three coaxial cables to maintain temperature stability (reduce the thermal exchange via the coaxial cables). Semirigid stainless steel coaxial cables were used inside the chamber and $\mathrm{Cu}$ coaxial cables were used outside the chamber. Fig. 3 (b) shows the enclosed box of the HTS MMIC circuit intergarted with the mini cryocooler, which has a dimension of approximately $350 \mathrm{~mm} \times 350 \mathrm{~mm} \times 250 \mathrm{~mm}$. This is a portable all-HTS microwave integrated receiver sub-system.

\section{EXPERIMENTAL RESUlTS OF THE PORTABLE HTS MMIC DOWN-CONVERTER}

The performance of one HTS MMIC down-converter integrated with the mini cryocooler was evaluated and the results at $55 \mathrm{~K}$ are presented here. The step-edge junction in this down-converter has an $R_{\mathrm{n}}$ value of $\sim 25 \Omega$ and unsuppressed $I_{\mathrm{c}}=15 \mu \mathrm{A}$ at $55 \mathrm{~K}$ (note that the $I_{\mathrm{c}}, R_{\mathrm{n}}$ values are different from the mixer reported in [11]). In this paper, we will not discuss in details the DC characteristics of the stepedge junction and their influence on the mixer performance such as the suppression of LO power $P_{\mathrm{LO}}$ on the junction $I_{\mathrm{c}}$ (i.e. $\left.\partial I_{\mathrm{C}} / \partial \mathrm{P}_{\mathrm{LO}}{ }^{1 / 2}\right)$ and the mixer conversion efficiency. More details of such discussion can be found in a recent paper [11]. 
A few measurement results of the HTS module integrated in the mini-cooler are presented here to demonstrate a working portable HTS receiver front-end. The cable losses have not been deducted from all the power levels shown in the graphs.

(a)

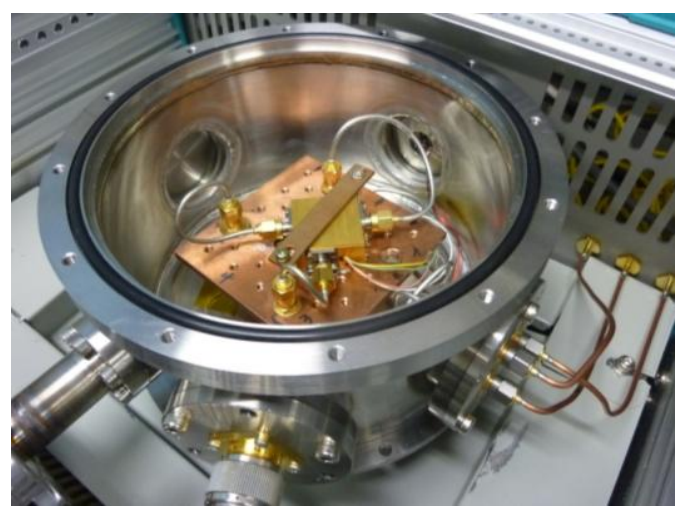

(b)

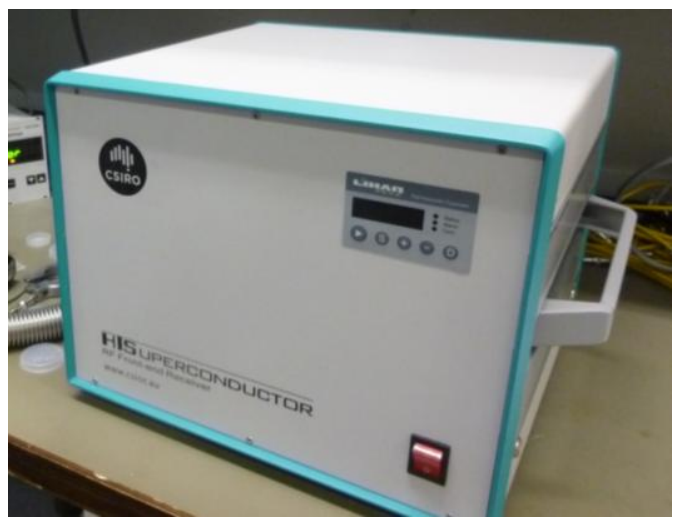

Fig. 3. The photographs of HTS down-converter module mounted in the vacuum chamber (upper picture) and the enclosed box that contains all parts (lower picture).

Figs. 4 and 5 are measured IF power outputs as the functions of the DC bias current, $I_{\mathrm{B}}$, and the LO power level. Two maxima of the IF output against the bias current $I_{\mathrm{B}}$ have been observed in all our measurements and explained in [11]. The working range of the LO power is quite broad but optimal point is around $-50 \mathrm{dBm}$ (include $\sim 5 \mathrm{~dB}$ cable loss). The required LO power is very small, which is one of the advantages of the Josephson mixer.

Fig. 6 shows the measured dynamic range and linearity of the IF output power for the MMIC Josephson down-converter. A highly linear relationship of the IF output versus the RF input power is displayed with a dynamic range over $40 \mathrm{~dB}$. The conversion efficiency estimated from $P_{\mathrm{IF}} / P_{\mathrm{RF}}$ less cable loss is approximately $-11 \mathrm{~dB}$, which is close to that $(\sim-10 \mathrm{~dB}$ at $55 \mathrm{~K}$ ) subsequently measured on the large two-stage PTC.

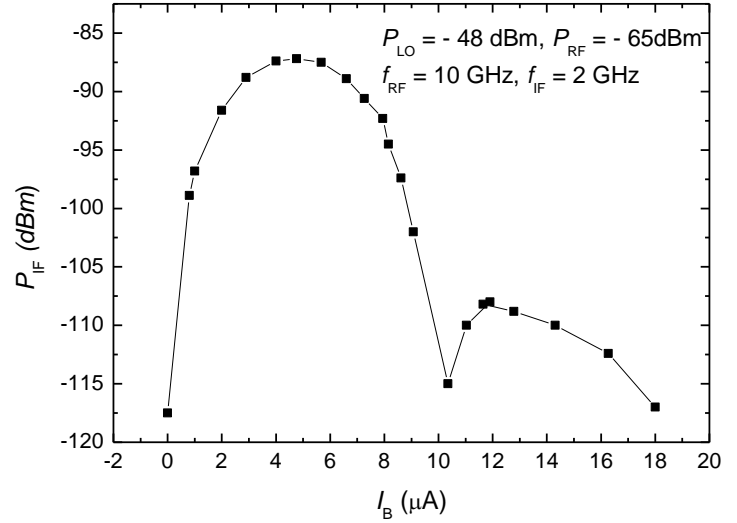

Fig. 4. IF output versus the bias current $I_{\mathrm{B}}$ at fixed RF frequency and LO power.

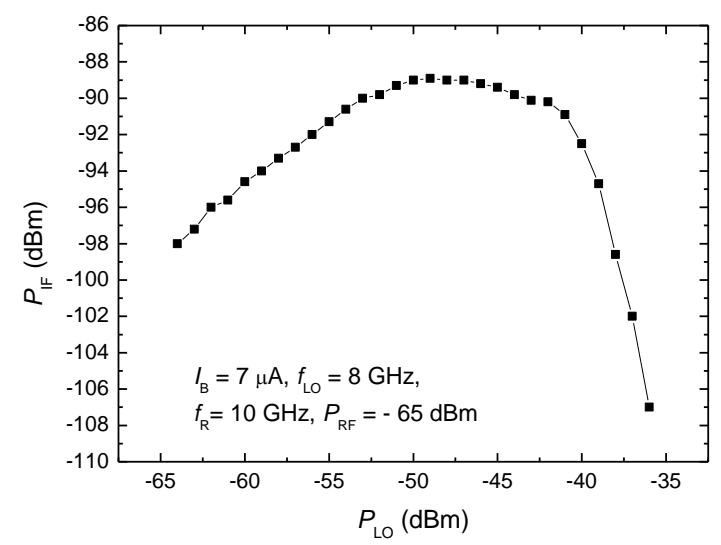

Fig 5. IF output as function of the LO power level at fixed frequency and RF power level.

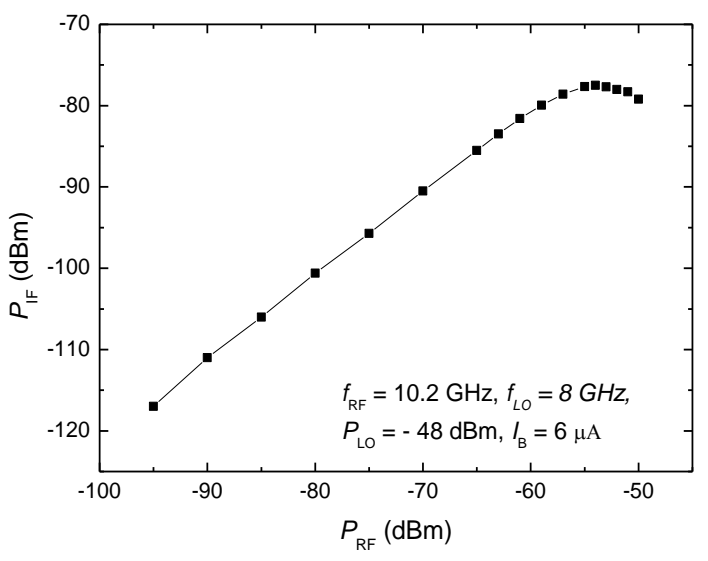

Fig. 6. IF output power versus input RF power for the MMIC down-converter 


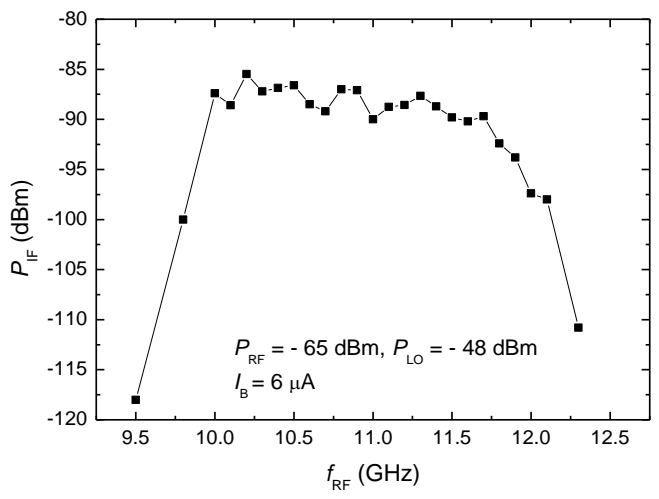

Fig. 7. Frequency response of the $10-12 \mathrm{GHz}$ MMIC frequency downconverter at $55 \mathrm{~K}$ cooled by the mini cryocooler.

Fig.7 is the frequency response of the HTS down-converter, of which the response shape is defined by the HTS bandpass filter, i.e. 10-12 $\mathrm{GHz}$ passband [3]. The same device was subsequently measured on the large PTC at the same temperature and the results were very close. Given the limited page length, only the frequency response of the device measured on the large PTC is given below for comparison (uncorrected cable loss). Slightly discrepancy in both conversion efficiency and frequency response is expected given different coaxial cables installed which affect the frequency characteristics and the cable loss estimation.

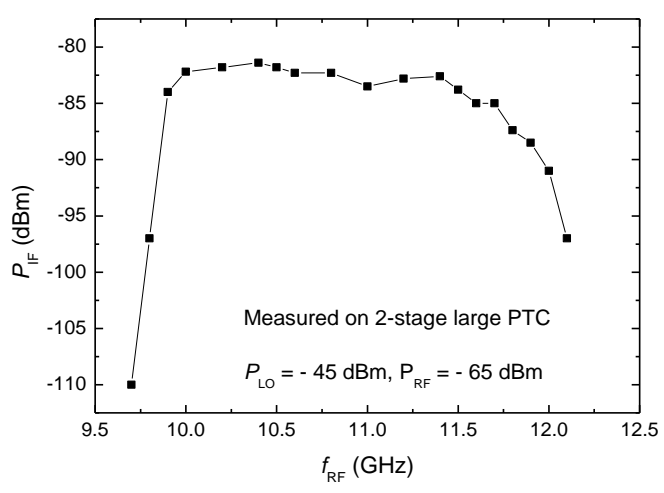

Fig. 8. Frequency response of the same MMIC down-converter but cooled by the large 2-stage PTC.

While only the measurement results of the HTS MMIC down-converter are presented here, we have also measured a number of other HTS junction-based microwave devices including the resistive-SQUID heterodyne oscillator and mixer $[5,9]$. The linewidth of the heterodyne oscillator operating on the mini cryocooler was measured and compared to that obtained on the large PTC. No additional linewidth broadening was observed at measured frequencies between $300 \mathrm{MHz}$ and $4 \mathrm{GHz}$ indicating minimum magnetic fields and EMI noise influence on the Josephson junction from the cryocooler. The results presented in this paper were obtained unshielded although a customer-designed mu-metal shield has been made and will be assessed in future work.

\section{CONCLUSIONS}

In this work, a portable HTS MMIC Josephson frequency down-converter operating on a commercial mini cryocooler is demonstrated. A compact unit was designed and used to house all cryocooler components, sample chamber and the MMIC HTS circuit. The HTS Josephson junction-based microwave circuit was operated successfully in the mini cryocooler unshielded and without observable performance degradation. This work demonstrated the potential of a portable all-HTS receiver front-end for application in wireless communications.

\section{ACKNOWLEDGMENT}

We thank LIHAN Thermoacoustic Technologies Co., LTD for supplying the mini cryocooler, providing customerdesigned modifications and fabricating the housing. We thank Mr Keith Leslie of CSIRO for useful discussion and help with magnetic field measurement, Jeina Lazar for HTS device fabrication, Chris Williams and Bill Vasilevski for coaxial cable and wire installation in the system.

\section{REFERENCES}

[1] J. S. Hong, M. J. Lancaster, D. Jedamzik, R. B. Greed and J. C. Mage, "On the performance of HTS microstrip quasi-elliptic function filters for mobile communications application", IEEE Trans. Microw. Theory Tech., 48, 1240-6200, 2000.

[2] X. Zhang, Q. Meng, F. Li, C. Li, S. Li, A. He, H. Li, and Y. He, "A 24pole high Tc superconducting filter for mobile communication applications", Superconduct. Sci. Technology., 19, S394-S397, 2006.

[3] D. D. Bai, Jia Du, Ting Zhang and Yusheng He, "Design of an HTS bandpass filter for an on-chip integrated Josephson down-converter", $J$. Appl. Phys., 114, 133906, 2013.

[4] T. Zhang, J. Du, Y. Jay Guo and X. W. Sun, "A compact HTS bandpass microstrip filter with novel coupling structure for on-chip integration", Physica C. 495, 69-73, 2013.

[5] J. Du, J. C. Macfarlane and L. Hao, "Noise temperature and linewidth of HTS R-SQUID GHz oscillators", Appl. Phys. Lett., 93, 033507, 2008.

[6] J. C. Macfarlane, J. Du J, R. Taylor and C. M. Pegrum, "Simulation and measurement of HTS Josephson heterodyne oscillator", IEEE Trans. Appl. Supercond., 19, 920-923, 2009.

[7] J. Du and J. C. Macfarlane, "Frequency down-conversion in the selfpumped HTS R-SQUID mixer", Electronics Letters, 47, 772-3, 2011.

[8] J. Du, J. C. Macfarlane, T. Zhang, Y. Cai and Y. J. Guo, "Self-Pumped HTS Josephson Heterodyne Tuneable Mixer", Supercond. Sci. Technol., 25, 025019, 2012.

[9] J. Du, T. Zhang, J. C. Macfarlane, Y. J. Guo, X. W. Sun, "Monolithic HTS heterodyne Josephson frequency down-converter", Appl. Phys. Lett. 100 262604, 2012.

[10] T. Zhang, J. Du, Y. J. Guo, X. W. Sun, "A High-Pperformance MMIC HTS Josephson Mixer", IEEE Trans Microwave amd Wireless Components Lett., 23 (8) 427-429, 2013.

[11] J. Du, D. D. Bai, T. Zhang, Y. J. Guo, Y. S. He and C. Pegrum, "Optimised Conversion Efficiency of a HTS MMIC Josephson Downconverter", Supercond. Sci. Technol. 27 105002, 2014.

[12] J. Oppenlaender, C. Haeussler, A. Friesch, J. Tomes, P. Caputo, T. Traeuble, and N. Schopohl, "Superconducting quantum interference filters operated in commercial miniature cryocoolers", IEEE Trans. Appl. Supercond. 15 936-939, 2005.

[13] T. Zhang, J. Du, Y. J. Guo and X. W. Sun, "Design and integration of HTS filters with a Josephson device," Supercond. Sci. Technol., 25 105014, 2012.

[14] C. P. Foley, E. E. Mitchell, S. K. H. Lam, B. Sankrithyan, Y. M. Wilson, D. L. Tilbrook and S. J. Morris, "Fabrication and characterisation of YBCO single grain boundary step-edge junctions", IEEE Trans. Appl. Supercond. 9 4281-4284, 1999.

[15] E. E. Mitchell and C. P. Foley, "YBCO Step-edge junctions with high IcRn”, Supercond. Sci. Technol. 23 065007, 2010. 